\title{
La ventilación no invasiva domiciliaria en pacientes con enfermedad pulmonar obstructiva crónica e hipercapnia podría asociarse con menor mortalidad, ingresos hospitalarios e intubaciones
}

Non-invasive home ventilation in patients with chronic obstructive pulmonary disease and hypercapnia could be associated with decreased mortality, hospital admissions, and intubations

\section{Comentado de:}

Wilson ME, et al. JAMA. 2020; 323:455-465. PMID: $32016309^{1}$

\section{Objetivo}

Evaluar si el uso de ventilación con presión positiva no invasiva (VPPNI) domiciliaria, en pacientes con enfermedad pulmonar obstructiva crónica (EPOC) e hipercapnia, se asocia a una menor mortalidad, ingresos hospitalarios, intubaciones y una mejor calidad de vida.

\section{Fuentes de datos}

Se realizó una búsqueda en MEDLINE, EMBASE, SCOPUS, el Registro Central Cochrane de ensayos controlados, la base de datos Cochrane de revisiones sistemáticas (Cochrane Database of Systematic Reviews) y el Centro de información nacional de guías de práctica clínica de los EE.UU. (National Guideline Clearinghouse), para artículos en inglés publicados hasta noviembre de 2019.

\section{Selección de estudios}

Se incluyeron ensayos clínicos controlados aleatorizados (ECA) y estudios observacionales analíticos que:

- Evaluaron a adultos (>18 años) con EPOC e hipercapnia que recibieron VPPNI domiciliaria, con dispositivos de presión positiva con dos niveles en la vía respiratoria (BiPAP) o de ventilación mecánica no invasiva (VMNI), al menos durante 1 mes;

- Compararon la VPPNI contra tratamiento usual u otro tipo de ventilación no invasiva;

- Reportaron los eventos de interés;

- Estuvieron escritos en inglés y publicados después de 1994.

Se excluyeron estudios observaciones de un solo grupo que midieron los resultados antes y después de la intervención.

\section{Selección de artículos y extracción de datos}

Fueron realizadas por dos revisores independientes. En caso de discrepancias entre los revisores, un tercer revisor resolvió el desacuerdo. El riesgo de sesgo fue evaluado mediante la herramienta Cochrane para evaluación del riesgo de sesgo de ECA y mediante elementos seleccionados de las escala NewcastleOttawa Scale para los estudios no aleatorizados.

\section{Medidas de desenlace}

Los desenlaces primarios fueron la mortalidad, las admisiones hospitalarias por todas las causas, la necesidad de intubación y la calidad de vida, al momento del seguimiento más prolongado.

\section{Resultados principales}

Esta revisión sistemática incluyó 21 ECA y 12 estudios observacionales, con un total 51.085 participantes. Se registraron 434 muertes y 27 pacientes necesitaron intubación.

El uso de cualquier dispositivo de VPPNI, comparado con la ausencia de soporte ventilatorio, se asoció con menor mortalidad, menor número de pacientes que necesitaron admisión hospitalaria por todas las causas y menor necesidad de intubación (ver Tabla 1), pero no se observaron diferencias significativas en la cantidad de ingresos hospitalarios o en la calidad de vida de los participantes.

El desempeño del uso de BiPAP, comparado con la ausencia de soporte ventilatorio, mostró resultados similares al análisis global, dado que la mayoría de los estudios habían evaluado esa intervención. En cambio, el uso de VMNI comparado con ningún dispositivo, se asoció con menos cantidad de hospitalizaciones, sin cambios en la mortalidad (tres estudios en total, confianza en la evidencia baja e insuficiente, respectivamente).

Tabla 1. Comparación de los eventos principales entre aquellos pacientes con enfermedad pulmonar obstructiva crónica que usaron cualquier dispositivo de ventilación con presión positiva no invasiva y ningún dispositivo. Notas: DR: diferencia de riesgo; IC: intervalo de confianza; OR: odds ratio. NR: no reportado \# Se reporta el riesgo relativo. *Se usaron tres tipos de escalas de calidad de vida, se reporta la diferencia estandarizada de medias con su IC $95 \%$.

\begin{tabular}{|l|c|c|}
\hline Desenlace & OR (IC 95\%) & DR (IC 95\%) \\
\hline Mortalidad & $0,65(0,48$ a 0,88$)$ & $-6,29(-11,5$ a $-1,08$ \\
\hline $\begin{array}{l}\text { Número de pacientes que necesitaron interna- } \\
\text { ción }\end{array}$ & $0,22(0,11$ a 0,43$)$ & $-35,26(-49,39$ a $-21,12)$ \\
\hline $\begin{array}{l}\text { Número de admisiones hospitalarias\# } \\
\text { Intubación }\end{array}$ & $0,75(0,52$ a 1,10$)$ & NR en la evidencia \\
\hline Calidad de vida* & $0,34(0,14$ a 0,83$)$ & $-8,02(-14,77$ a $-1,28)$ \\
\hline
\end{tabular}


El efecto beneficioso en la mortalidad se encontró cuando la VPPNI fue iniciada en pacientes con EPOC estable, pero no cuando esta terapia fue iniciada después de una exacerbación.

Por otro lado, en el análisis de sensibilidad en el que sólo se incluyeron los ECA, no se encontró ninguna diferencia estadísticamente significativa.

Aproximadamente uno de cada cinco pacientes que utilizaron la VPPNI presentaron efectos adversos leves, por inconvenientes cutáneos, oculares, oronasales, gastrointestinales o intolerancia de la máscara.

\section{Conclusiones}

En este meta-ánálisis, los pacientes con EPOC e hipercapnia, el BiPAP domiciliario, en comparación con ningún dispositivo, se asoció con un menor riesgo de mortalidad, ingreso hospitalario por cualquier causa e intubación. Sin embargo, no hubo diferencias significativas en la calidad de vida. La VMNI se asoció con menor riesgo de admisión hospitalaria, sin cambios en la mortalidad.

Fuente de financiamiento/Conflicto de interés de los autores: Este estudio fue financiado por la Agencia de Investigación en Salud y Calidad del Departamento de Salud y Servicios Humanos de los EE.UU.

\section{Comentario}

El fundamento de usar la VPPNI en pacientes con EPOC se basa en que este dispositivo puede disminuir el trabajo respiratorio y mejorar la mecánica ventilatoria ${ }^{2}$. La VPPNI en pacientes con EPOC e insuficiencia respiratoria aguda secundaria a una exacerbación ha demostrado disminuir la mortalidad, el número de intubaciones y de complicaciones ${ }^{3}$. Pero la evidencia en EPOC estable con hipercapnia es controvertida.

Aunque el meta-análisis evaluado sugiere beneficios con el uso de BiPAP con respecto a la mortalidad, ingresos hospitalarios y riesgo de intubaciones, hay que considerar las limitaciones del estudio. En primer lugar, la confianza en la evidencia encontrada fue leve a moderada, debido en parte al riesgo de sesgo de los estudios incluidos (por problemas en el cegamiento de la intervención y posible riesgo de conflictos de interés, en los ECA, y por falta de claridad en los métodos de selección de pacientes, aspectos relacionados con el pronóstico, y la dificultad para evaluar posibles conflictos de interés, en los estudios observacionales), y no se pudo descartar la posibilidad de sesgo de publicación. En segundo término, cuando se analizaron solamente los ECA no hubo diferencias estadísticamente significativas en los eventos de interés. Además, el umbral de la presión parcial de dióxido de carbono en la sangre arterial $\left(\mathrm{PaCO}_{2}\right)$ para el inicio de la VPPNI fue muy variable, y las recomendaciones a nivel mundial son distintas en este punto ${ }^{4-6}$. Finalmente, se utilizaron diferentes modalidades ventilatorias, dispositivos e intensidades de presión de soporte, lo que determina que los estudios hayan sido clínicamente heterogéneos.

\section{Conclusiones del comentador}

Si bien el estudio sugiere que los pacientes con EPOC estable e hipercapnia se beneficiarían con el uso de VPPNI domiciliaria, el nivel de evidencia fue de bajo a moderado con limitaciones importantes. Principalmente la variabilidad de los modos de ventilación posibles, el uso de distintos dispositivos y los diferentes umbrales de $\mathrm{PaCO}_{2}$ para iniciar el tratamiento hacen que los resultados sean difíciles de trasladar a la práctica clínica diaria. Se necesitan más estudios para apoyar la indicación de este tratamiento.

Horacio Matías Castro [ Sección Neumonología, Servicio de Clínica Médica, Hospital Italiano de Buenos Aires. matias.castro@hospitalitaliano.org.ar ]

Castro HM. La ventilación no invasiva domiciliaria en pacientes con enfermedad pulmonar obstructiva crónica e hipercapnia podría asociarse con menor mortalidad, ingresos hospitalarios e intubaciones. Evid Actual Pract Ambul. 2020;23(2):e002048. Comentado de: Wilson ME, et al. Association of Home Noninvasive Positive Pressure Ventilation With Clinical Outcomes in Chronic Obstructive Pulmonary Disease: A Systematic Review and Meta-analysis. JAMA. 2020; 323:455-465. PMID: 32016309

\section{Referencias}

1. Wilson ME, Dobler CC, Morrow AS, et al. Association of Home Noninvasive Positive Pressure Ventilation With Clinical Outcomes in Chronic Obstructive Pulmonary Disease A Systematic Review and Meta-analysis. JAMA. 2020;323(5):455-465. Available from: 10.1001/jama.2019.22343.

2. Coleman JM, Wolfe LF, Kalhan R. Noninvasive Ventilation in Chronic Obstructive Pulmonary Disease. Annals of the American Thoracic Society. 2019;16(9):1091-1098. Available from: 10.1513/annalsats.201810-657cme;https://dx.doi.org/10.1513/annalsats.201810-657cme.

3. Osadnik CR, Tee VS, Carson-Chahhoud KV, et al. Non-invasive ventilation for the management of acute hypercapnic respiratory failure due to exacerbation of chronic obstructive pulmonary disease. Cochrane Database of Systematic Reviews. 2017;7(7):CD004104. Available from: 10.1002/ 14651858.CD004104.pub4.

4. Windisch W, Walterspacher S, Siemon K, et al. Guidelines for Non-Invasive and Invasive Mechanical Ventilation for Treatment of Chronic Respiratory Failure. Published by the German Society for Pneumology (DGP). Pneumologie. 2010;64(10):640-652. Available from: 10.1055/s-0030-1255558.

5. National Institute for Health and Care Excellence. Chronic obstructive pulmonary disease in over 16s: diagnosis and management; 2018. Available from: https://www.nice.org.uk/guidance/NG115 [Last access: 2020-05-06].

6. McKim DA, Road J, Avendano M. Home mechanical ventilation: A Canadian Thoracic Society clinical practice guideline. Can Respir J. 2011;18(4):197-216. Available from: 0.1155/2011/139769. 